\title{
Carreau-Casson fluids flow and heat transfer over stretching plate with internal heat source/sink and radiation
}

\author{
Hajar F. Ismael * \\ Department of Mathematics, University of Zakho, Duhok, Kurdistan Region, Iraq
}

\section{A R T I C L E I N F O}

\section{Article history:}

Received 8 February 2017

Received in revised form

29 April 2017

Accepted 19 May 2017

\section{Keywords:}

Carreau-casson MHD fluid

Heat transfer

Shooting method

\begin{abstract}
A B S T R A C T
In this research, Carreau-Casson Fluids flow under the effect of energy transfer with internal heat source/sink and radiation over a stretching sheet are being analyzed and investigated. Shooting method with the help of 4order Runge-Kutta (RK4) integration technique is applied on governing equation of fluid flow and heat equation. The effect of dimensionless governing parameters on velocity, thermal profiles along with the friction factors and local Nusselt numbers is showed graphically and numerically. Different physical interesting parameters on the fluid velocity and heat equation are described visually and numerically.
\end{abstract}

(c) 2017 The Authors. Published by IASE. This is an open access article under the CC BY-NC-ND license (http://creativecommons.org/licenses/by-nc-nd/4.0/).

\section{Introduction}

The fluid flow and heat flux within a sheet film is attention for the understanding, analyze and the design of different heat exchangers and chemical processing equipment. The multi applications of this study include wire and fiber coating, aerodynamic extrusion of plastic plate, reactor fluidization, polymer plate and food processing, and cooling of transpiration. Crane (1970) was the first author believed to examine the fluid flow on a stretching sheet in view of an application to the process of polymer extrusion from a slit. Later, more authors Gupta and Gupta (1977), Carragher and Crane (1982) and Howell et al. (1997) investigated various aspects of same problems, such as the heat transfer, mass and momentum equation of a semi-infinite fluid layer driven by a continuous stretching plate.

The non-uniform heat source/sink influence on the fluid flow and thermal boundary layer from an unsteady stretching plate through a quiescent fluid medium extending to infinity is investigated. The velocity profile and heat field are solved numerically using the Chebyshev finite difference method (Tsai et al., 2008). Abel et al. (2009) studied a mathematical model of MHD flow and heat exchange to a horizontal laminar plate, the flow of a thin liquid film and subsequent heat exchange from the stretching surface is investigated with the aid of

\footnotetext{
* Corresponding Author.

Email Address: hajar.ismael@uoz.edu.krd

https://doi.org/10.21833/ijaas.2017.07.003

2313-626X/C) 2017 The Authors. Published by IASE.

This is an open access article under the CC BY-NC-ND license

(http://creativecommons.org/licenses/by-nc-nd/4.0/)
}

similarity transformation, the transformation enables to transform the unsteady boundary layer equations to a system of non-linear ordinary differential equations. Nandeppanavar et al. (2012) analyzed the effects of viscous dissipation; nonuniform heat source/sink, magnetic field, and thermal radiation on temperature characteristics over an unsteady stretching sheet of a thin liquid film are discussed.

Baag et al. (2016) studied MHD flow analysis on a stretching sheet in a porous medium using DTMPade' and Numerical Methods (shooting method), the influences of various interesting parameters like as magnetic parameter, permeability parameter, and the power index is discussed. Yousif et al. (2016) investigated numerical simulation for a thin liquid sheet over unsteady stretching shoot by using homotopy perturbation technique which found that when increasing both of the Darcy number and the unsteadiness parameter will decrease the thickness of the thin liquid film. Magneto hydrodynamic Casson fluid with heat transfer in stretching sheet is investigated by using shooting method with help of RK-4 and analyzed physical interesting parameter Ali et al. (2017).

Several authors Vajravelu and Roper (1999), Vajravelu (2001), Liu (2004) and Sajid and Hayat (2008) examined the layer boundary with heat exchange problem with a linear, power-law or exponentially surface velocity in a stretching plate and a uniform or various surface heat condition. In this paper, the main objective is to undertake the study of the Carreau-Casson fluid flow and energy transfer over an unsteady stretching surface with the study, the effect of viscous dissipation, thermal radiation and non-uniform heat source/sink under 
the effect of a magnetic field. In order to solve governing equation, we use shooting method with help of 4-order Runge-Kutta (RK4) integration scheme to find the approximate solution of nonlinear ordinary differential equations that covers the MHD boundary layer Carreau-Casson flow and a thermal boundary layer of Carreau-Casson fluid in the presence of a magnetic parameter.

\section{Mathematical model}

Assume two-dimensional continuity equation, momentum equation, and energy equation that cover incompressible viscous MHD fluid with electrically conducting. The transitive applied magnetic field $B_{0}$ is normal to the stretching sheet are formulated. The Navier-Stocks equation with heat equation that governing the problem are (Eqs. 1-3)

$\mathrm{u}_{\mathrm{x}}+\mathrm{v}_{\mathrm{y}}=0$,

$\mathrm{uu}_{\mathrm{x}}+\mathrm{vu}_{\mathrm{y}}=\mathrm{v}\left(1+\frac{1}{\beta}\right) \mathrm{u}_{\mathrm{yy}}+\mathrm{v} \frac{(3 n-3)}{2} \Gamma^{2} \mathrm{u}_{\mathrm{yy}} \mathrm{u}_{\mathrm{y}}{ }^{2}-\sigma \frac{\mathrm{B}^{2}(\mathrm{x})}{\rho} \mathrm{u}-$ $\frac{\mathrm{v}}{\mathrm{K}} \mathrm{u}$,

$\mathrm{uT}_{\mathrm{x}}+\mathrm{vT}_{\mathrm{y}}=\frac{\mathrm{K}}{\rho \mathrm{c}_{\mathrm{p}}} \mathrm{T}_{\mathrm{yy}}+\frac{\mu}{\rho \mathrm{c}_{\mathrm{p}}} \mathrm{u}_{\mathrm{y}}^{2}-\frac{1}{\rho \mathrm{c}_{\mathrm{p}}}\left(\mathrm{q}_{\mathrm{r}}\right)_{\mathrm{y}}+\frac{\mathrm{q}^{\prime \prime \prime}}{\rho \mathrm{c}_{\mathrm{p}}}$

Here $u$ and $v$ represents the velocity components along $x$ and y directions, respectively. $v, \rho$ and $\sigma$ are the kinematic viscosity, density and electrical conductivity of the fluid; Casson fluid by $\beta$ and time constant by $\Gamma$. Also, T, $K$ and $c_{p}$ are the temperature, thermal diffusivity and specific heat, respectively. Where the external electric field is ignored and transverse magnetic $B(x)$ of uniform strength is defined as:

$\mathrm{B}(\mathrm{x})=\mathrm{B}_{0} \mathrm{x}^{\mathrm{n}-\frac{1}{2}}$

$q_{r}$ and $q^{\prime \prime \prime}$ is the radiative heat flux and non-uniform heat source/sink of the fluid, defined as:

$\mathrm{q}_{\mathrm{r}}=-\frac{4 \sigma^{*}}{3 \mathrm{k}^{*}}\left(4 \mathrm{TT}_{\infty}{ }^{3}-3 \mathrm{~T}_{\infty}{ }^{4}\right)$

$\mathrm{q}^{\prime \prime \prime}=\frac{\mathrm{u}_{\mathrm{w}} \alpha}{2 \mathrm{~L} v}\left(\mathrm{~A}^{*}\left(\mathrm{~T}_{\mathrm{w}}-\mathrm{T}_{\infty}\right) \mathrm{f}^{\prime}+\mathrm{B}^{*}\left(\mathrm{~T}-\mathrm{T}_{\infty}\right)\right)$

where $\sigma^{*}$ and $\mathrm{k}^{*}$ are the Stefan-Boltzmann constant and the mean absorption coefficient respectively.

The associative boundary conditions that cover Eqs. 1-3, could be written as (Eqs. 4 and 5):

$\mathrm{u}(\mathrm{x}, \mathrm{y})=\mathrm{ax}, \mathrm{v}(\mathrm{x}, \mathrm{y})=0, \mathrm{~T}=\mathrm{T}_{\mathrm{w}}(\mathrm{x})$ at $\mathrm{y}=0$

$\mathrm{u}=0, \quad \mathrm{~T}=\mathrm{T}_{\infty}$ as $\mathrm{y} \rightarrow \infty$

where $\mathrm{T}_{\mathrm{w}}=\mathrm{T}_{\infty}+\frac{\mathrm{c} \xi^{-\frac{3}{2}} \mathrm{x}^{2}}{2 \mathrm{v}}$, and $T_{0}$ is a heating or cooling temperatures. For solving this problem, the continuity equation, momentum equation and energy equation, by below transformation with the help of stream function $\psi$ which are defined

$\psi=\sqrt{\mathrm{av}} \mathrm{xf}(\eta) ; \quad \eta=\sqrt{\frac{\mathrm{a}}{\mathrm{v}} \mathrm{y}}$
$\mathrm{g}=\frac{\mathrm{T}-\mathrm{T}_{\infty}}{\mathrm{T}_{\mathrm{w}}-\mathrm{T}_{\infty}}, \mathrm{T}=\mathrm{T}_{\infty}-\mathrm{T}_{\text {ref }} \operatorname{axg} \mathrm{g}(\eta)$

$\mathrm{u}=\frac{\partial \psi}{\partial \mathrm{y}}$ and $\mathrm{v}=-\frac{\partial \psi}{\partial \mathrm{x}}$

are converted into non-dimensional governing equations.

Apply transformations to Eqs. 1-5, Eq. 1, automatically will be identified, and the e Eqs. 2-5, are converted like as (Eqs. 7-10):

$$
\begin{aligned}
& \left(1+\frac{1}{\beta}\right) \mathrm{f}_{\eta \eta \eta}+\mathrm{ff}_{\eta \eta}-\mathrm{f}_{\eta}{ }^{2}-\mathrm{Mf}_{\eta}-\lambda \mathrm{f}_{\eta}+ \\
& \frac{(3 \mathrm{n}-3)}{2} \mathrm{We}_{\eta \eta}{ }^{2} \mathrm{f}_{\eta \eta \eta}=0 \\
& (1+\mathrm{R}) \frac{1}{\operatorname{Pr}} \mathrm{g}_{\eta \eta}-2 \mathrm{f}_{\eta} \mathrm{g}+\mathrm{fg}_{\eta}+E c \mathrm{f}_{\eta \eta}{ }^{2}+\frac{1}{\operatorname{Pr}}\left(\mathrm{A}^{*} \mathrm{f}_{\eta}+\mathrm{B}^{*} \mathrm{~g}\right)=
\end{aligned}
$$

the boundary conditions, become

$\mathrm{f}(0)=0, \mathrm{f}_{\eta}(0)=1, \mathrm{~g}(0)=1$ at $\eta=0$

$\mathrm{f}_{\eta}(\infty) \rightarrow 0, \quad \mathrm{~g}(\infty) \rightarrow 0$, at $\eta \rightarrow \infty$

$\mathrm{R}=\left(\frac{4 \sigma^{*} \mathrm{~T}_{0}^{3}}{\alpha \mathrm{k}^{*}}\right), \operatorname{Pr}=\frac{\mathrm{c}_{\mathrm{p}} \mu}{\alpha}, \mathrm{Ec}=\frac{\mathrm{a}^{2} \mathrm{x}^{2}}{\mathrm{c}_{\mathrm{p}}\left(\mathrm{T}_{\mathrm{w}}-\mathrm{T}_{\infty}\right)^{\prime}}$

$\mathrm{We}=\frac{\Gamma^{2} \mathrm{a}^{3} \mathrm{x}^{2}}{\mathrm{v}}, \mathrm{M}=\frac{\sigma \mathrm{B}_{0}{ }^{2}}{\rho \mathrm{a}}, \lambda=\frac{\mathrm{v}}{\mathrm{Ka}}$,

for physics and engineering interesting the skinfriction coefficient, the Nusselt number, and the reduced Sherwood numbers are given as:

$\mathrm{Cf}_{\mathrm{x}}=2 \operatorname{Re}_{\mathrm{x}}^{-\frac{1}{2}} \mathrm{f}^{\prime \prime}(0)$,

$\mathrm{Nu}_{\mathrm{x}}=-2 \operatorname{Re}_{\mathrm{x}}^{\frac{1}{2}} \mathrm{~g}^{\prime}(0)$

where, $\operatorname{Re}_{\mathrm{x}}=\frac{\mathrm{u}_{\mathrm{sx}}}{\mathrm{v}}$ is the local Renolds number and $\mathrm{u}_{\mathrm{s}}=\mathrm{ax}$ is stretching velocity.

\section{Numerical solution}

To solve and investigate interesting physical parameters and numbers, firstly we decomposed the original ODEs into a system of 1-order ordinary differential equals by letting (Eqs. 11 and 12):

$\omega_{1}=f, \omega_{2}=f^{\prime}, \omega_{3}=f^{\prime \prime}, \omega_{4}=g, \omega_{5}=g^{\prime}$

which gives

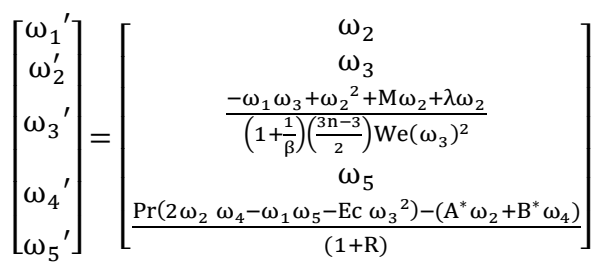

and the corresponding initial conditions are

$\left[\begin{array}{l}\omega_{1} \\ \omega_{2} \\ \omega_{3} \\ \omega_{4} \\ \omega_{5}\end{array}\right]=\left[\begin{array}{c}0 \\ 1 \\ \varphi_{1} \\ 1 \\ \varphi_{2}\end{array}\right]$

The dimensionless for velocity and temperature Eqs. 11 and 12 with the boundary conditions (13) 
and (14) have been solved numerically by shooting method with fourth-order Runge-Kutta (RK4) integration scheme.

To solve Eq. 15 with Eq. 16 with its initial value problem by shooting method with the help of 4order Runge-Kutta (RK4) integration technique, we need to find the unknown values for $\varphi_{1}$ and $\varphi_{2}$. The unknown initial values for $f^{\prime \prime}(0)$ and $g^{\prime}(0)$ are founded and the 4-order Runge-Kutta integration technique is used to catch the solution. The maximum magnitude of $\eta \rightarrow \infty$, to each interesting parameter is determined when the values of unknown boundary conditions at $\eta=0$ do not vary to a successful loop with absolute error smaller than $10^{-5}$.

\section{Discussion and results}

An appropriate similarity transformation is used to reduce or transform the governing partial differential equations of Carreau-Casson fluids flow equation and heat problem into a system of nonlinear ordinary differential equations. The boundary layer problem is solved by using shooting method with help of RK-4 scheme, all figures are plotted for $\beta=0.01, M=1, \lambda=1, \operatorname{Pr}=1, R=1, A^{*}=0.01$, $B^{*}=0.01, E c=0.01, W e=1$ and $n=2$ on $f^{\prime}(\eta)$ and $g(\eta)$.

The behavior of Casson fluid Flow on velocity profile is examined in Fig. 1, for different value of the $\beta$ parameter; from this figure it is obvious that Casson the parameter was a decrees coefficient on axial velocity.

Figs. 2 and 3 demonstrate the influence of Weissenberg number $W e$ and constant $\mathrm{n}$ on the velocity profile for different values for both of them, it is clear that both of them have different effect in comparisons with $\mathrm{M}, \lambda$ and Casson fluid $\beta$, which one could say $W e$ and $\mathrm{n}$ are increase coefficient of boundary value problems.

The influence of the Hartmann number $\mathrm{M}$ and the porosity parameter $\lambda$ on the axial velocity are showed in Figs. 4 and 5 for different values of interesting parameters, it presented graphically that the value of boundary layer thickness decreases with increasing in magnitude parameter of both $M$ and $\lambda$.

In Figs, 6, 7, 8, 9, and 10, we analyze the physical parameter on heat transfer, Fig. 6 and Fig. 7 depicts the influence of the Prandtl number (Pr) and $B^{*}$ on the temperature profiles, it is clear that an increase in the values of Pr and temperature dependent heat source/sink $\mathrm{B}^{*}$ contributes to the tinning of the thermal boundary layer.

The effect of Eckert number (Ec), thermal radiation parameter $\mathrm{R}$ and space dependent heat source/ sink $A^{*}$ on the temperature profile are presented in Figs. 8, 9, and 10, increases the rate of physical parameters which causes the thermal boundary layer of fluid to increase.

Local skin friction coefficient and local Nusselt number for various values of physical parameters which are discussed in this boundary layer problem with heat transfer are displayed in a Table 1.

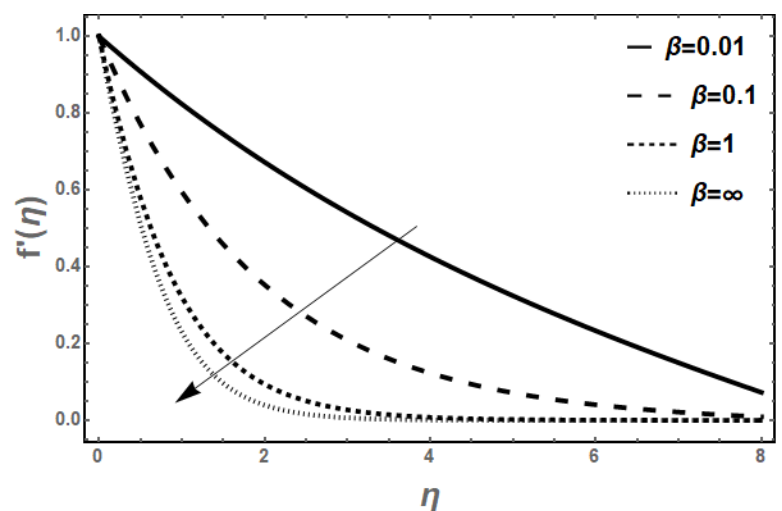

Fig. 1: Plotted for different value of $\beta$ on $f^{\prime}(\eta)$

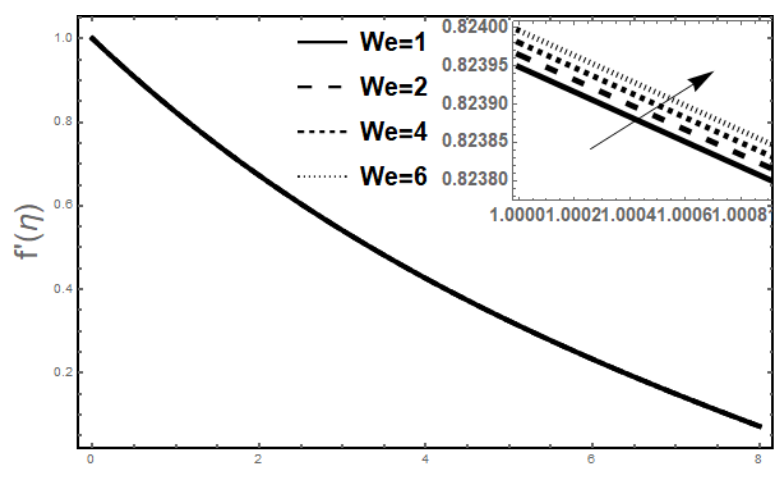

$\eta$

Fig. 2: Plotted for different value of $W e$ on $f^{\prime}(\eta)$

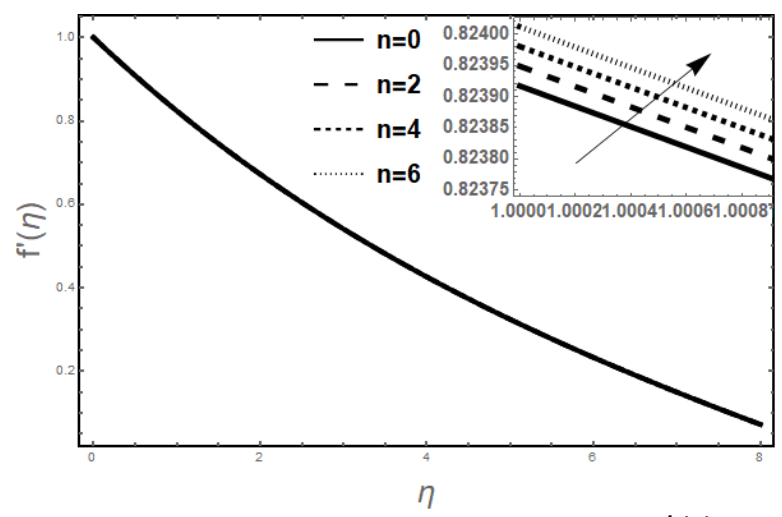

Fig. 3: Plotted for different value of $n$ on $f^{\prime}(\eta)$

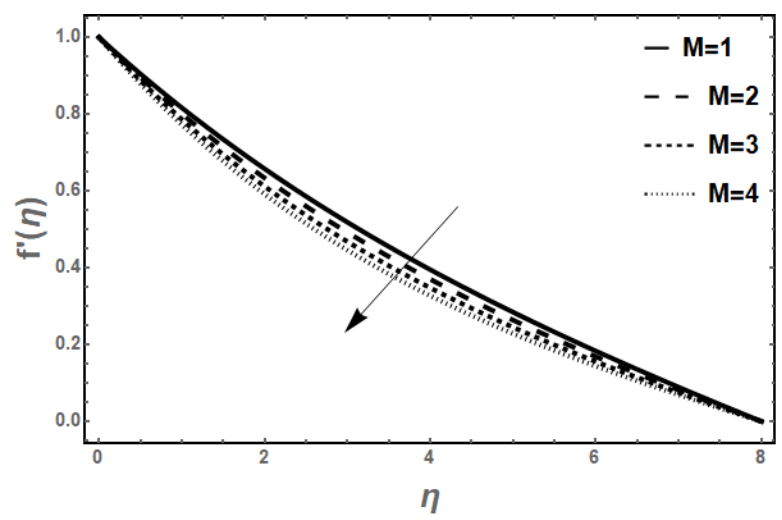

Fig. 4: Plotted for different value of $M$ on $f^{\prime}(\eta)$

\section{Conclusion}

In this study, MHD boundary layer of CarreauCasson fluids under the effect of heat transfer is examined. The governing nonlinear ordinary differential equations are solved numerically by 
adopting shooting method. The impact of all the interesting physical parameters and numbers is illustrated with the help of figures.

Table 1: Comparison for finding the value of $f^{\prime \prime}(0)$ and $g^{\prime}(0)$ using different magnitudes of parameter

\begin{tabular}{|c|c|c|c|c|c|c|c|c|c|c|c|c|}
\hline Number & $\beta$ & $\mathbf{M}$ & $\lambda$ & Pr & $\mathbf{R}$ & $\boldsymbol{A}^{*}$ & $B^{*}$ & Ec & We & $\mathrm{n}$ & $-f^{\prime \prime}(0)$ & $-g^{\prime}(\mathbf{0})$ \\
\hline 1 & 0.01 & 1 & 1 & 1 & 1 & 0.01 & 0.01 & 0.01 & 1 & 2 & 0.189993419391 & 1.0727097653768993 \\
\hline 2 & 0.1 & & & & & & & & & & 0.517814489754 & 0.9867916601201487 \\
\hline 3 & 1 & & & & & & & & & & 1.037055768657 & 0.8169969968913097 \\
\hline 4 & $\infty$ & & & & & & & & & & 1.20589290958 & 0.738962979656889 \\
\hline 5 & 0.01 & 1 & & & & & & & & & 0.1972172266419 & 1.0705081886486492 \\
\hline 6 & & 2 & & & & & & & & & 0.2173924713642 & 1.0655490455520165 \\
\hline 7 & & 3 & & & & & & & & & 0.23645455203 & 1.060823503358435 \\
\hline 8 & & 4 & & & & & & & & & 0.2545347055335 & 1.0563063001739408 \\
\hline 9 & & 1 & 1 & & & & & & & & 0.1972172266419 & 1.0705081886486492 \\
\hline 10 & & & 2 & & & & & & & & 0.2173924713642 & 1.0655490455520165 \\
\hline 11 & & & 3 & & & & & & & & 0.236454552033 & 1.060823503358435 \\
\hline 12 & & & 4 & & & & & & & & 0.2545347055335 & 1.0563063001739408 \\
\hline 13 & & & 1 & 1 & & & & & & & 0.1899934193913 & 1.0727097653768993 \\
\hline 14 & & & & 2 & & & & & & & 0.1899934181811 & 1.5426454105977554 \\
\hline 15 & & & & 3 & & & & & & & 0.1899934197456 & 1.9023968469896122 \\
\hline 16 & & & & 4 & & & & & & & 0.1972172267519 & 2.203408538525036 \\
\hline 17 & & & & 1 & 1 & & & & & & 0.1972172266419 & 1.0705081886486492 \\
\hline 18 & & & & & 2 & & & & & & 0.197217226644 & 0.8642952120733189 \\
\hline 19 & & & & & 3 & & & & & & 0.1972172266538 & 0.7411901525460889 \\
\hline 20 & & & & & 4 & & & & & & 0.1972172266332 & 0.6571358006148859 \\
\hline 21 & & & & & 1 & 0.01 & & & & & 0.1899934196074 & 1.0727097715266354 \\
\hline 22 & & & & & & 0.1 & & & & & 0.189993419405 & 1.0276166767485204 \\
\hline 23 & & & & & & 1 & & & & & 0.1899934196074 & 0.5766857887362731 \\
\hline 24 & & & & & & 3 & & & & & 0.189993419607 & 0.4253828633661532 \\
\hline 25 & & & & & & 0.01 & 0.01 & & & & 0.1899934196074 & 1.0727097715266354 \\
\hline 26 & & & & & & & 1 & & & & 0.1899934196074 & 0.7963240489558914 \\
\hline 27 & & & & & & & 2 & & & & 0.18999341961 & 0.22878992403139686 \\
\hline 28 & & & & & & & -1 & & & & 0.197217226493 & 1.2897690886156585 \\
\hline 29 & & & & & & & 0.01 & 0.01 & & & 0.189993419391 & 1.0727097653768993 \\
\hline 30 & & & & & & & & 2 & & & 0.1899934194188 & 1.0400558237474355 \\
\hline 31 & & & & & & & & 5 & & & 0.18999341941 & 0.99082877775889022 \\
\hline 32 & & & & & & & & 8 & & & 0.1899934194179 & 0.941601730068325 \\
\hline 33 & & & & & & & & 0.01 & 1 & & 0.1899934196074 & 1.0727097715266354 \\
\hline 34 & & & & & & & & & 2 & & 0.189970952313 & 1.0727147485989246 \\
\hline 35 & & & & & & & & & 4 & & 0.1899485059144 & 1.0727197217049194 \\
\hline 36 & & & & & & & & & 6 & & 0.1899260803738 & 1.0727246908492336 \\
\hline 37 & & & & & & & & & 1 & 0 & 0.197265109168 & 1.0704975337056755 \\
\hline 38 & & & & & & & & & & 2 & 0.1972172266419 & 1.0705081886486492 \\
\hline 39 & & & & & & & & & & 4 & 0.1971694405504 & 1.0705188250873112 \\
\hline 40 & & & & & & & & & & 6 & 0.1971217505198 & 1.0705294430865875 \\
\hline
\end{tabular}

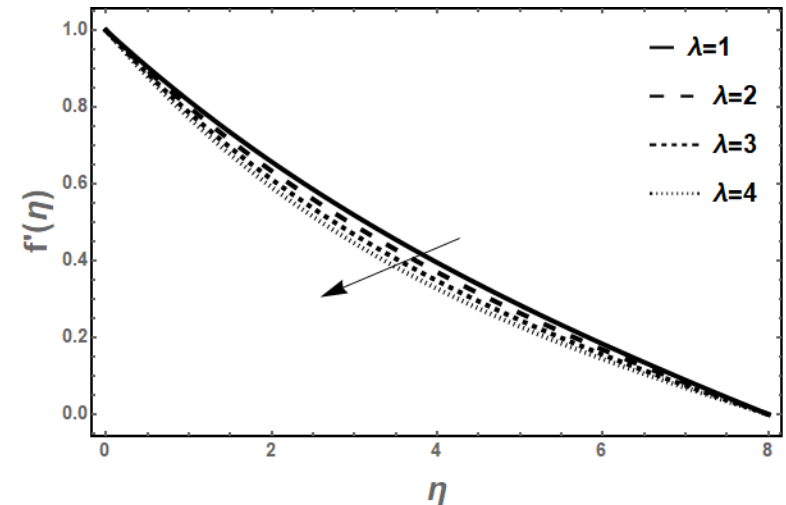

Fig. 5: Plotted for different value of $M$ on $f^{\prime}(\eta)$

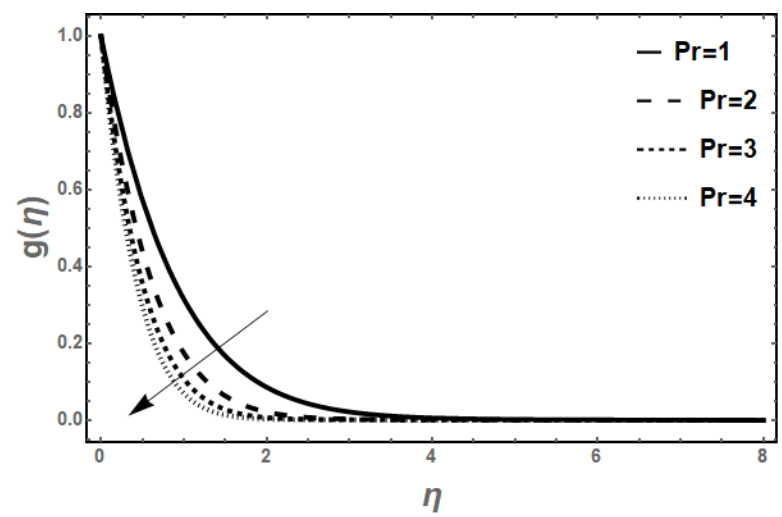

Fig. 6: Plotted for different value of Pr on $g(\eta)$

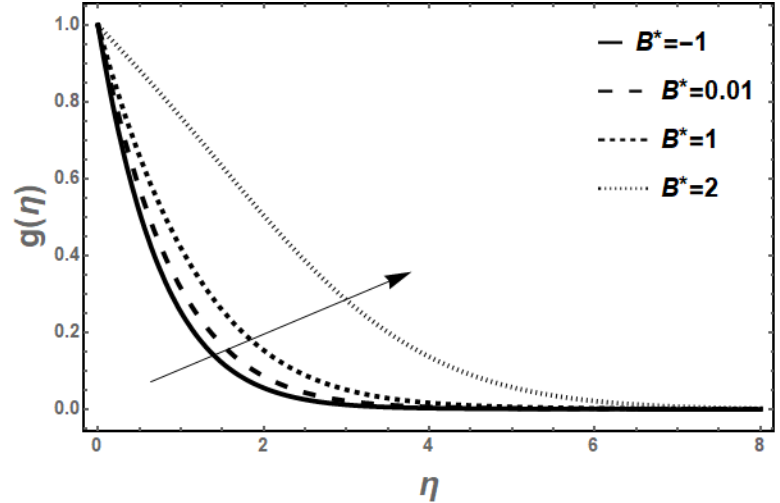

Fig. 7: Plotted for different value of $B^{*}$ on $g(\eta)$

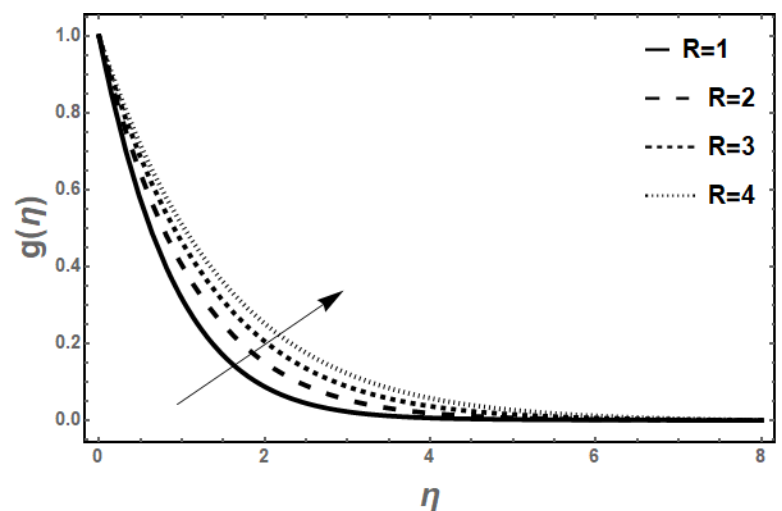

Fig. 8: Plotted for different value of $R$ on $g(\eta)$ 


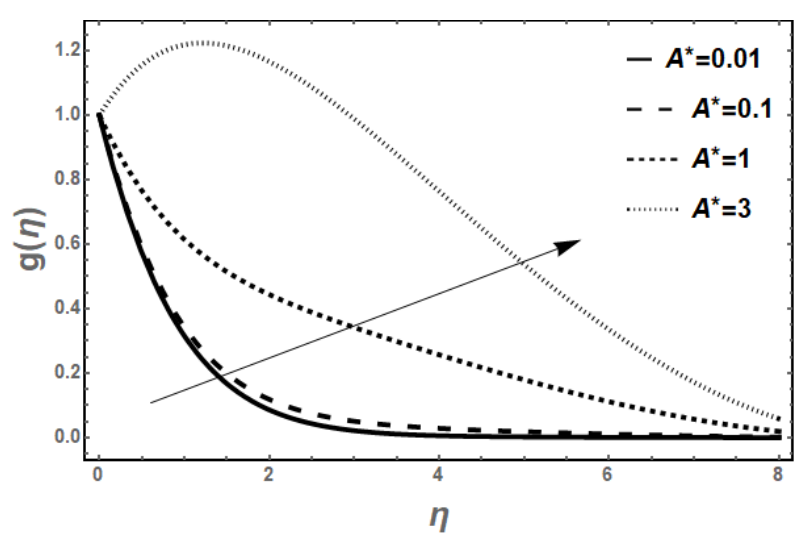

Fig. 9: Plotted for different value of $A^{*}$ on $g(\eta)$

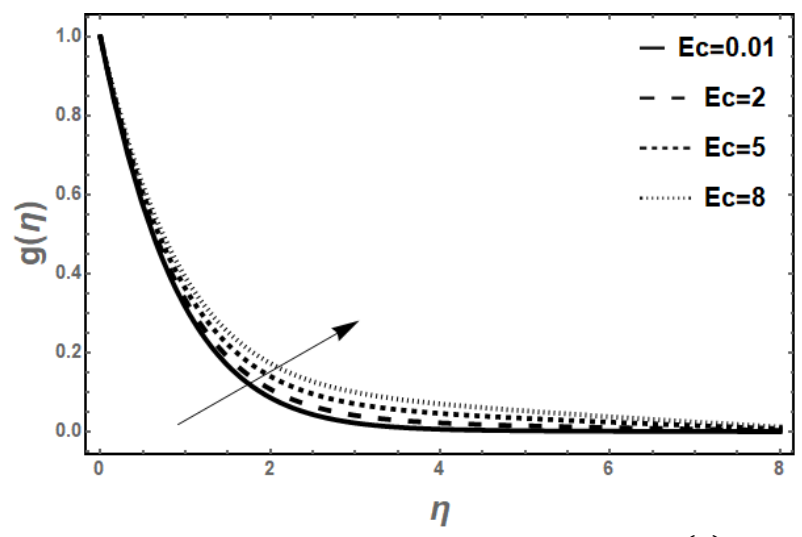

Fig. 10: Plotted for different value of $E c$ on $g(\eta)$

\section{References}

Abel MS, Mahesha N, and Tawade J (2009). Heat transfer in a liquid film over an unsteady stretching surface with viscous dissipation in presence of external magnetic field. Applied Mathematical Modelling, 33(8): 3430-3441.

Ali KK, Ismael HF, Mahmood BA, and Yousif MA (2017). MHD Casson fluid with heat transfer in a liquid film over unsteady stretching plate. International Journal of Advanced and Applied Science, 4(1): 55-58.

Baag S, Mishra S, Dash G, and Acharya M (2016). Entropy generation analysis for viscoelastic MHD flow over a stretching sheet embedded in a porous medium. Ain Shams
Engineering Journal. Available online at: http://www.sciencedirect.com/science/article/pii/S2090447 915002099

Carragher P and Crane L (1982). Heat transfer on a continuous stretching sheet. ZAMM-Journal of Applied Mathematics and Mechanics [Zeitschrift für Angewandte Mathematik und Mechanik], 62(10): 564-565.

Crane LJ (1970). Flow past a stretching plate. Zeitschrift für angewandte Mathematik und Physik (ZAMP), 21(4): 645-647.

Gupta PS and Gupta AS (1977). Heat and mass transfer on a stretching sheet with suction or blowing. Canadian Journal of Chemical Engineering, 55(6): 744-746.

Howell TG, Jeng DR, and De Witt KJ (1997). Momentum and heat transfer on a continuous moving surface in a power law fluid. International Journal of Heat and Mass Transfer, 40(8): 1853 1861.

Liu IC (2004). Flow and heat transfer of an electrically conducting fluid of second grade over a stretching sheet subject to a transverse magnetic field. International Journal of Heat and Mass Transfer, 47(19): 4427-4437.

Nandeppanavar MM, Vajravelu K, Abel MS, Ravi S, and Jyoti H (2012). Heat transfer in a liquid film over an unsteady stretching sheet. International Journal of Heat and Mass Transfer, 55(4): 1316-1324.

Sajid M and Hayat T (2008). Influence of thermal radiation on the boundary layer flow due to an exponentially stretching sheet. International Communications in Heat and Mass Transfer, 35(3): 347-356.

Tsai R, Huang KH, and Huang JS (2008). Flow and heat transfer over an unsteady stretching surface with the non-uniform heat source. International Communications in Heat and Mass Transfer, 35(10): 1340-1343.

Vajravelu K (2001). Viscous flow over a nonlinearly stretching sheet. Applied Mathematics and Computation, 124(3): 281288.

Vajravelu K and Roper T (1999). Flow and heat transfer in a second-grade fluid over a stretching sheet. International Journal of Non-Linear Mechanics, 34(6): 1031-1036.

Yousif MA, Mahmood BA, Ali KK, and Ismael HF (2016). Numerical simulation using the homotopy perturbation method for a thin liquid film over unsteady stretching. International Journal of Pure and Applied Mathematics, 10(2): 289-300. 